\title{
DINAMICA Y MANEJO DE POBLACIONES DE MALAS HIERBAS
}

\author{
MILAGROS S. SAAVEDRA ${ }^{2}$
}

\section{RESUMEN}

El presente trabajo es una revisión de la modelización de estudios de dinámica de poblaciones de malas hierbas, que fue presentada como ponencia en el XVIII Congresso Brasileiro de Herbicidas e Plantas Daninhas en 1991, Brasília. Partiendo de la población, como unidad básica de los ecosistemas agrarios, se relacionan diferentes modelos de dinámica incluyendo el fenómeno de competencia intra específica, la evolución del banco de semillas y aplicaciones a la asociación cultivo y mala hierba. A través de los diagramas de ciclos vitales, que relacionan los estados funcionales y los procesos demográficos, se llega al establecimiento del modelo matricial.

Palabras claves: competencia intra-específica, banco de semillas.

\section{ABSTRACT \\ Dynamics and management of weed populations}

This literature review is a report about the study model of weed population dynamics, which was presented at the XVIII Congress of the Brazilian Weed Science Society, held in Brasilia, Brazil, 1991. Starting from population as the basic block of farm ecosystems, different models of dynamics are related, including the phenomenon of intraspecific

\section{ORGANIZACION DE UN ECOSISTEMA AGRARIO}

El medio agrario es un sistema complejo en el que intervienen diversos componentes: cultivo, malas hierbas, microorganismos, clima, suelo, etc., los cuales están relacionados entre sí. El problema planteado por las malas hierbas dentro de este sistema puede estructurarse en 4 niveles (Fig. 1): individuo, población, comunidad y agro-ecosistema en su conjunto (Fernández-Quíntanilla, 1990).

Una población es un grupo de individuos de una misma especie que habitan en un área determinada. Se considera a la población en un medio limitado convencionalmente, en

1 Recebido para publicação em 20/07/93 e na forma revisada em 28/12/93. 2 Departamento de Protección Vegetal, Centro de Investigación y Desarrollo Agrario de Córdoba, Servicio de Investigación Agraria, Junta de Andalucia, Apdo. 4240, 14080 Cordoba, España. competition, the evolution of seed banks, and applications to the weed/crop association. Through life cycle diagrams, which relates the functional status and the demographic processes, it is possible to achieve a matricial model.

Additional index words: intraspecific competition, seed bank.

cuanto a espacio y a tiempo, y por tanto separado de otros grupos por barreras naturales o artíficiales.

Esta característica de "aislamiento" de los individuos que componen la población, respecto de otros individuos de otras poblaciones, es particularmente importante, porque va

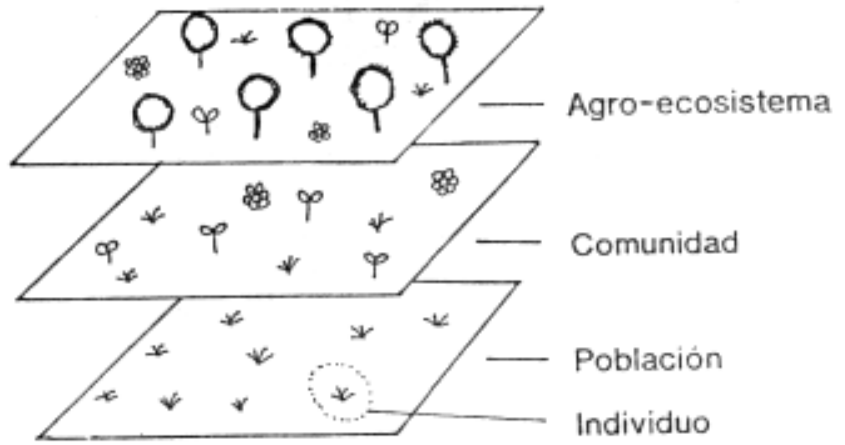

FIG. 1 - Niveles de organización de un ecosistema agrario. (Fernández-Quintanilla, 1990). 
a permitir estudiar una población, cómo varía en el tiempo y qué factores influyen en su tamano y evolución. Y todo ello utilizando métodos relativamente sencillos.

\section{EL ESTUDIO DE LAS POBLACIONES COMO UNIDAD BASICA DE LOS ECOSISTEMAS AGRARIOS}

Cuando se trata de estudiar un ecosistema en su conjunto resulta muy laborioso porque hay muchas especies y muchos factores implicados. En un medio natural encontramos muchos individuos de diversas especies en equilibrio más o menos estable dentro de su entorno; el estudio de las poblaciones que lo componen es bastante complejo por la diversidad de especies, en cambio tiene de ventaja que a lo largo de los anos es mucho más estable.

Por el contrario el medio agrario, el campo de cultivo, es un medio artificial, y en ciertos aspectos más simple, en el que intervienen: especies introducidas (cultivo y malas hierbas), factores biológicos, prácticas culturales, aplicaciones de herbicidas, etc. El hombre es el mayor responsable de las modificaciones producidas por las prácticas agrícolas:

- Ha establecido un sistema de cultivo, que Ie permite controlar sin "esfuerzo aparente" o "gasto adicional" muchas de las especies de malas hierbas presentes.

- La presión sobre el medio reduce el número de especies del ecosistema, más concretamente de malas hierbas.

- La modificación de las condiciones naturales, regadío por ejemplo, permiten la introducción de especies procedentes de otros ecosistemas.

Como consecuencia:

- Determinadas especies de malas hierbas quedan "aisladas" dentro del sistema de cultivo establecido. El problema agronómico de control de malas hierbas se reduce respecto al ecosistema natural. Desde el punto de vista económico, se debe fundamentalmente a pocas especies dominantes, incluso en algunos casos a una sola especie que no es controlada mediante las prácticas habituales de cultivo.

- $\quad$ Los individuos de estas especies son susceptibles deser estudiados como POBLACION.

\section{DINAMICA DE UNA POBLACION Y COSECHA}

Los rendimientos de un cultivo dependen en gran medida de la presencia de las malas hierbas. En muchísimas ocasiones se ha relacionado la producción con medidas de abundancia: biomasa y densidad. Por tanto el tamano de la población es importante porque repercute en las producciones.

La población de una determinada especie arvense varía en el tiempo. En un momento dado se puede cuantificar. El estado de partida puede ser semillas, plántulas, plantas, etc.; pero normalmente se determina el número de semillas o propágulos vegetativos en suelo, por ser el estado menos fluctuante y más independiente de variaciones ambientales anuales.

Hay poblaciones de especies que se estabílizan en el tiempo, adoptando una estrategia de permanencia, mientras que otras descienden, $\mathrm{u}$ otras son oportunistas y crecen al verse afectadas por algún factor. En la figura 2 se indican alguns modelos de crecimiento de poblaciones.

Diversos factores regulan el tamano de una población y éstos pueden clasificarse en tres tipos: dependientes de la densidad, independientes de la densidad y de manejo.
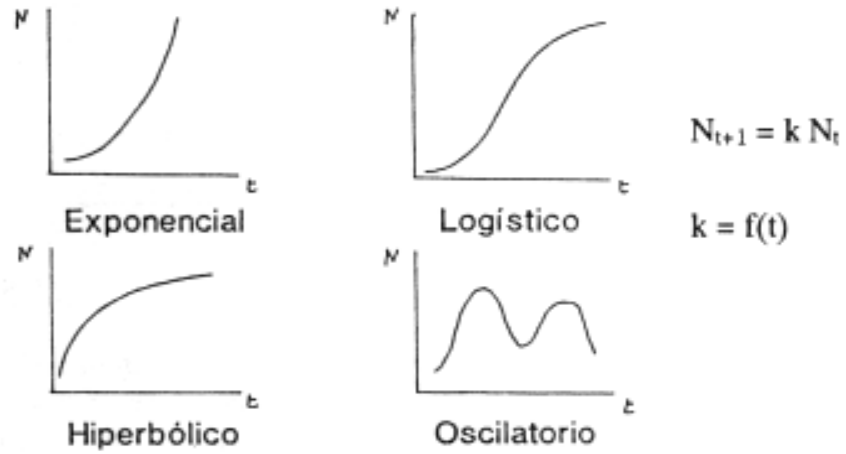

FIG. 2 - Modelos de crecimiento de poblaciones. $\mathbf{t}=$ tiempo, $\mathbf{N}=$ tamaño de la población, $\mathbf{k}=$ parámetro, tasa de crecimiento.

\section{Factores dependientes de la densidad:}

Existen una serie de mecanismos de adaptación de las malas hierbas a los recursos disponibles del medio. Cuando la densidad de plantas es baja los recursos son amplios y las plantas crecen vigorosamente y se reproducen con profusión. En cambio si la densidad es alta los recursos son limitados para cada planta y con ello su supervivencia y capacidad reproductiva, es decir, la población se autorregula y tiende a estabilizasse. Un ejemplo de ésto se indica en la figura 3.
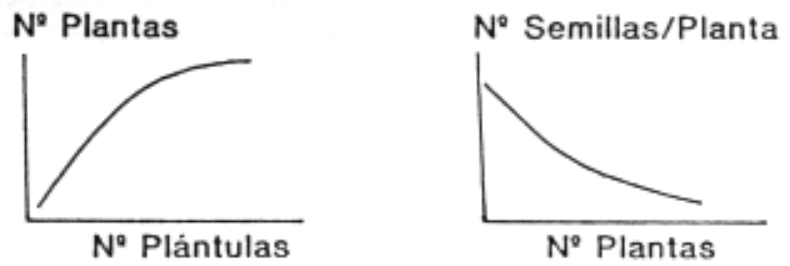

FIG. 3 - Influencia de la densidad en la mortalidad de plantas y en la producción de semilla.

\section{- Factores independientes de la densidad:}

Otros factores externos a la población actúan disminuyendo el número de plantas o su capacidad reproductiva, como por ejemplo los accidentes meteorológicos: heladas, sequía, ataques de patógenos o depredadores, etc.

\section{- Factores antrópicos:}

El hombre a su vez contribuye de forma directa a regular la población de malas hierbas actuando directamente sobre la población o modificando las condiciones del medio.

Los estudios de dinámica de poblaciones tienen por objeto determinar el tamano de las poblaciones a lo largo del tiempo y de los factores que influyen en dicho tamano. La aplicación más inmediata de estos estudios es determinar 
cuantitativamente la influencia sobre los rendimientos de Ias cosechas en anos sucesivos, así como predecir la influencia que tienen determinadas prácticas agrícolas sobre el tamano de esa población, a efectos de conseguir regular las poblaciones y obtener los mayores rendimientos económicos con el menor esfuerzo y menor riesgo de producir alteraciones en el medio ambiente.

\section{DEMOGRAFIA DE LAS POBLACIONES: CICLO DE VIDA, ESTADOS FUNCIONALES Y PROCESOS DEMOGRAFICOS}

Demografía es el estudio de los aspectos cuantitativos del nacimiento, desarrollo, reproducción y muerte de una población. Los estudios demográficos se realizan mediante el establecimiento de estados funcionales (semilla, plántula, adulto, banco de semillas, etc.) y los procesos demográficos que regulan el paso de un estado funcional al siguiente (nascencia de plántulas, supervivencia, reproducción, etc.).

La separación de estados funcionales permite analizar y cuantificar más fácilmente los factores que intervienen en los procesos demográficos $\mathrm{y}$, por tanto, cuales son los que más contribuyen al crecimiento o descenso de una población. En las figuras 4a y 4b se muestran ejemplos de ciclos demográficos de una especie monocárpica y de una especie perenne con 5 estados funcionales respectivamente.

\section{LA ESQUEMATIZACION DEL PROBLEMA: CICLO DE VIDA Y MODELOS}

El estudio de la dinámica de poblaciones y de los procesos demográficos se realiza estableciendo modelos. Un modelo es la representación simplificada de la realidad, y el nivel de complejidad de un modelo dependerá del propósito para el que se construya.

Los objetivos que persigue un modelo son: - Organizar los conocimientos acerca de un fenómeno.
- Identificar los aspectos desconocidos de ese fenómeno.
- Ayudara conocer sistemascomplejos.
- Predecir la respuesta de un sistema al modificar si- tuaciones.
- Ayudar a la toma de decisiones en el manejo de los sistemas.

Los modelos se pueden clasificar en conceptuales (lingüísticos o diagramáticos) y funcionales (matemáticos). En los estudios demográficos y dinámica de poblaciones son muy utilizados los modelos matemáticos y el diagrama de Forrester (Fig. 5). En el diagrama se indicará el ciclo de vida de la planta en forma esquematizada, detallando los procesos que tienen lugar y anotando los factores que influyen en cada proceso. Posteriormente se pasará a cuantificar la población y los procesos aplicando los modelos matemáticos.

Los modelos matemáticos

Un modelo matemático pueden ser:

- Dinámíco (contiene la variable tiempo) o estático.
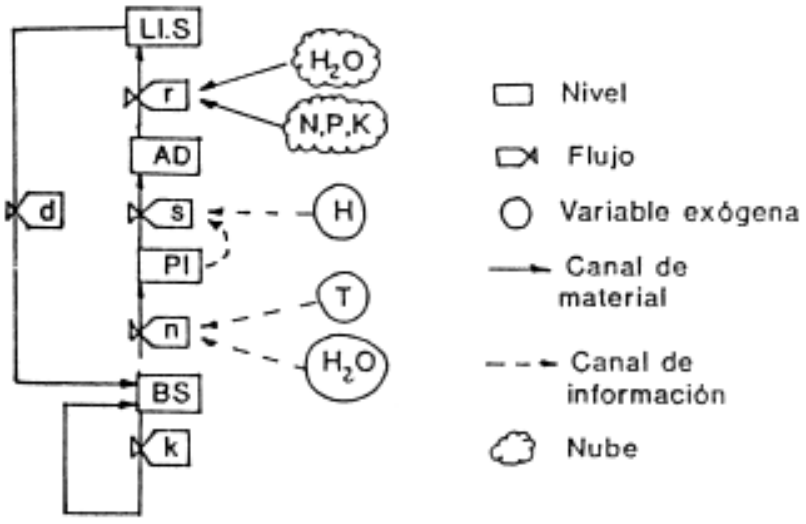

FIG. 5 - Diagrama de Forrester. LI.S = lluvia de semillas; $\mathrm{AD}=$ adultos $; \mathbf{P I}=$ plántulas $; \mathbf{B S}=$ banco de semillas; $\mathbf{r , s , n , d , k ~ = ~ p a r a ́ m e t r o s ~ d e m o g r a ́ f i c o s ~}$ que relacionan cuantitativamente dos estados funcionales consecutivos; $\mathbf{H}=$ herbicida; $\mathbf{T}=$ temperatura.

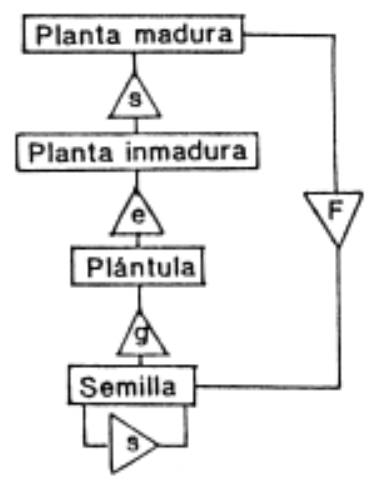

a. ESPECIE MONOCARPICA

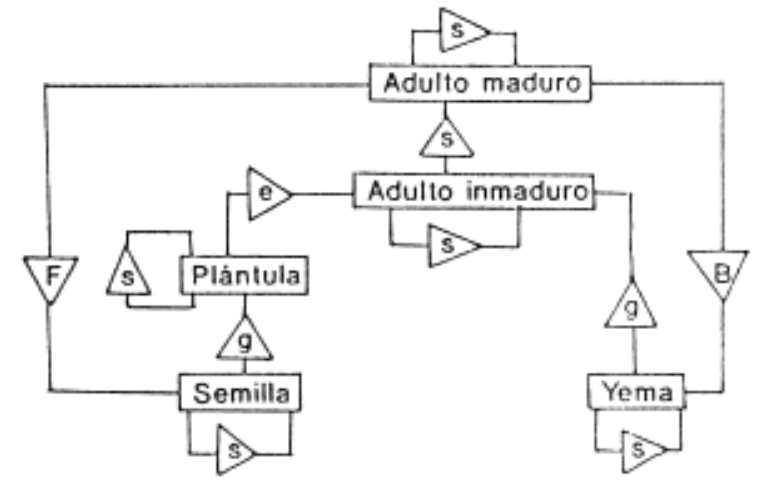

b. ESPECIE PERENNE CON 5 ESTADOS FUNCIONALES

FIG. 4 - Ciclo de vida, estados funcionales y procesos demográficos. $s$ = probabilidad de supervivencia entre estado y estado; $\mathrm{g}=$ probabilidad de germinación de semillas; e = Probabilidad de establecimiento de plántulas; $\mathbf{F}=$ fecundidad de semillas; $\mathbf{B}$ = fecundidad de yemas. 
- Determinista (realiza las predicciones sin asociación a distribuciones de probabilidad) o estocástico (contiene elementos al azar o distribuciones de probabilidad dentro del modelo y predice valores esperados junto con la varianza).

- Empírico (describe sin explicar cómo funciona el sistema) o mecanístico (describe incluyendo los mecanismos que explican cómo funciona el sistema).

\section{- Biológicoso aplicativos}

El establecimiento de un modelo matemático en biología implica, como es lógico, la conjunción de 2 disciplinas fundamentales: biología y matemáticas. En el caso de las ciencias agrárias, y en particular en nuestro caso de Ias malas hierbas, tendremos que anadir la agronomía, y un cuarto que es la informática, indispensable cuando el sistema es complejo. Para el caso de modelos mecanísticos es asimismo fundamental conocer los aspectos fisiológicos relacionados con el sistema.

Los modelos matemáticos contribuyen de muy diversas formas a las ciencias agrarias (France, 1988):

- Las hipótesis biológicas expresadas en términos matemáticos proporcionan una descripción cuantitativa de conocimientos de los problemas biológicos.

- Permiten considerar un proceso completo y detectar las lagunas de conocimiento y estudiar nuevas ideas y aproximaciones experimentales.

- $\quad$ Facilitan al agricultor el conocimiento más fácil de un problema complejo de investigación.

- Los beneficios prácticos de una modificación de los sistemas pueden ser evaluados y estimular su adopción.

- Facilitan el diseno experimental.

- Con ayuda de un computador permiten ser utilizados por los agricultores, extensionistas y por los propios investigadores de forma fácil.

Ejemplos de modelos que permiten predecir cambios en una población de malas hierbas los encontramos: en Aarts (1986) para Galium aparine y, en relación a la eficacia de control, en Firbank y Watkinson (1986) para Agrostemma githago. Otros autores utilizan los modelos poblacionales para el estudio del control económico a largo plazo, por ejemplo Avena fatua y Alopecurus myosuroides (Cousens, 1986).

\section{METODOS DE ESTUDIO DE LA DINAMICA DE POBLACIONES}

Una vez esquematizado el funcionamiento de la población y, dependiendo de los recursos económicos de que se disponga para llevar a cabo los trabajos de investigación, se establecerá la metodología de estudio de la dinámica de una población. Se pueden considerar 3 niveles de complejidad: estudios a largo plazo, estudios demográficos y análisis mecanísticos.

\section{Estudios a largo plazo}

Es el método más simple y comprende únicamente el seguimiento de la población en un estado funcional concreto a lo largo de los anos. Para estos estudios hay que elegir un estado funcional que indique lo mejor posible las tendencias a largo plazo de la población. Generalmente se utiliza el contenido de propágulos vegetativos y/o semillas del suelo. Estudios de este tipo han sido hechos por Fernánde z Quintanilla et al., (1984) para Avena ludoviciana (Fig. 6).

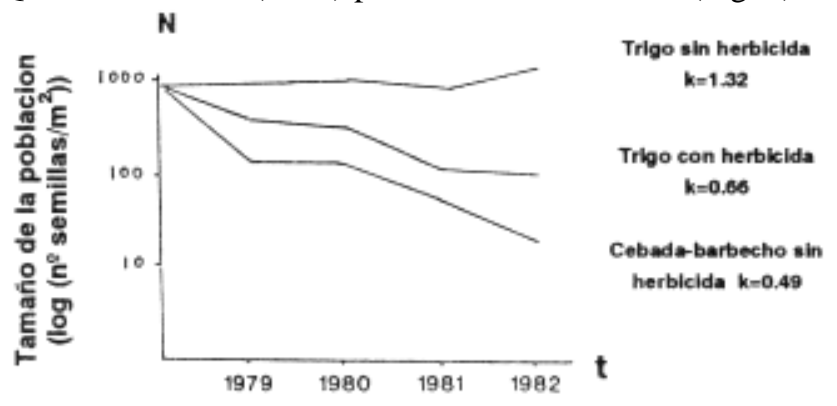

FIG. 6 - Dinámica de Avena sterilis subsp. ludoviciana. (Fernández-Quintanilla, 1984).

Las ventajas de este método son que:

Noprecisa el establecimiento de un modelo.

Essimplederealizar.

Los resultados son realistas.

Los inconvenientes son que:

- Requiere muchos anos de estudio y, por tanto, es lento.

- No analiza los efectos de factores influyentes. -

Por consiguiente tiene un bajo valor predictivo.

Las aplicaciones principales son:

- Evaluaciones a largo plazo de programas de control. Validaciónde los modelos desimulación.

\section{Estudios demográficos}

Fueron introducidos en malherbología por Sager y Mortimer (1976). Pueden ser estudios simples que interrelacionan estados funcionales, como los de Palma et al. (1990), López-Granados y García-Torres (1990); o pueden ser estudios más complejos, pudiéndose dividir por ejemplo el ciclo de vida para las distintas cohortes (Fernández-Quintanilla, 1988; Mortimer et a!., 1980) o analizar rotaciones JiménezHidalgo et a!. (1990). El proceso es más complicado cuando se analizan rotaciones. También se pueden estudiar las fases activas (vegetativas) sólas o junto con las pasivas (semillas en suelo).

En el caso de especies perennes el estudio puede llegar a ser muy complejo, puesto que al tamano de la población contribuyen varios estados funcionales al mismo tiempo, que además pueden ser activos simultáneamente o no. Alguns autores han considerado estos aspectos en su conjunto, como por ejemplo para Agropyron repens (Mortimer, 1983).

Las ventajas de estos métodos son principalmente que:

- Pueden ser utilizados, aunque con ciertas limitaciones, para descripción, análisis y predicción de la evolución de la población.

- No son tan complejos como los estudios mecanicistas. Tienen un valor analítico muy superiora los estudios a largo plazo. 
Los inconvenientes son que:

- Requieren un esfuerzo considerable.

- Los resultados son válidos bajo las condiciones de estudio.

- El valor predictivo es mediano, tanto mayor cuanto más detallado sea el estudio.

Las aplicaciones son:

- Comprensióndel funcionamiento de la población.

- Identificación del estado o proceso crítico que influye especialmente en la regulación del tamaño de la población.

Estos estudios pueden abordarse en uno o en pocos ensayos de campo en los que se cuantifican todos los estados funcionales y su interrelación, con lo cual se consigue en varios anos tener al mismo tiempo los parâmetros demográficos y los resultados finales del sistema, es decir, el resultado de un estudio a largo plazo y en consecuencia su validación.

Pero también pueden llevarse a cabo determinando cada uno de los parâmetros demográficos que interrela cionan dos estados funcionales consecutivos en distintos tipo de ensayo. Este último procedimiento presenta algunas ventajas como son:

- Utilizar fincas de agricultores o pequenos ensayos puntuales que permiten obtener datos más exhaustivos en distintas condiciones ambientales.

- $\quad$ No es necesario muestrear un mismo ensayo durante varios anos, ahorrando el realizar un diseño más o menos complejo, pero que ocuparía una gran superficie.

- $\quad$ Permite introducir en el esquema estados "puente" que asocien dos estados consecutivos.

- Los resultados pueden ser más rep resentativos de una zona o comarca, porque el número de variantes que permite analizar es mayor que si se restringen a pocos ensayos muy detallados.

- $\quad$ No se altera la población al obtener las muestras de las parcelas elementales.

- Permiten introducir en el sistema variables adicionales, como por ejemplo la reducción en el número de semillas producidas con distintos tratamientos herbicidas.

- Permiten avanzar en el sistema introduciendo factores ambientales y ]legar a un análisis mecanístico.

- Permite realizar el estudio en condiciones más naturales, sin los inconvenientes de restringirse a las condiciones de un ensayo en concreto.

- Se evita el riesgo de perder un ensayo de campo complejo por circunstancias anómalas imprevistas, por ejemplo una granizada, un ano agrícola con condiciones climáticas atípicas en la localidad, posibilidad de que haya una plaga, errores humanos, fallos en una siembra o nascencia del cultivo, etc.

- $\quad$ Se evita el pisoteo de las parcelas por los muestreos y las manipulaciones realizadas en una misma campana.

- $\quad$ Se facilita el estudio de las rotaciones largas.

- Posibilita la introducción de medias y varianzas y aproximasse a modelos estocásticos.
Como inconvenientes importantes tenemos que:

- $\quad$ Elnúmero deensayos puede ser excesivo.

- $\quad$ Requiere hacer un estudio a largo plazo para poder realizar la validación de los resultados.

\section{Estudios mecanísticos}

Los estudios demográficos ofrecen una visión general de los procesos que regulan el tamaño de las poblaciones, sin embargo no dan suficiente información sobre los factores que influyen en los cambios de la población.

En los estudios mecanísticos el ciclo de vida se descompone en distintos estados de crecimiento y desarrollo de la planta. El número de procesos involucrados es muy alto, por lo que es necesario determinar o intuir "a priori" cuales tienen una mayor influencia en el tamaño de la población. Se consideran los procesos fisiológicos y ecológicos y se establecen las relaciones funcionales entre los factores biológicos y medioambientales. Por ejemplo, en el caso de Avena sterilis (Fernández-Quintanilla, 1988) se ha demostrado que la germinación de semillas es uno de los procesos importantes que determinan el tamaño de la población. La tasa de germinación está influenciada por factores internos medioambientales y culturales (Fig. 7).

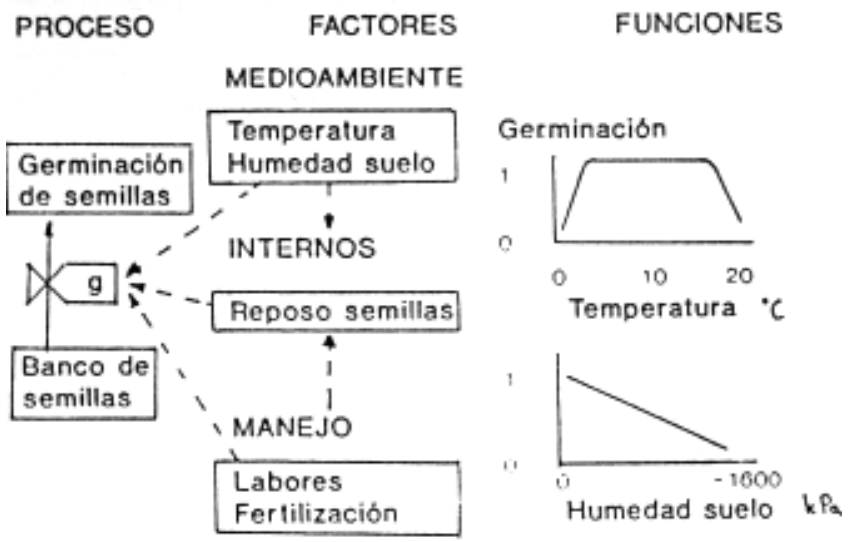

FIG. 7 - Germinación de Avena sterilis subsp. ludoviciana. (Fernández-Quintanilla, 1988).

Estos estudios mecanísticos han sido utilizados en muy pocos casos hasta ahora, pero ofrecen una considerable capacidad predictiva potencial. El mayor inconveniente es la necesidad de unos recursos humanos y económicos importantes, puesto que no se trata sólo de poner de manifiesto la relación entre un factor ambiental y un proceso, sino de cuantificar el efecto estableciendo las relaciones funcionales.

\section{Limitaciones de los estudios de dinâmica y demografia de las poblaciones}

Los estudios de dinâmica y demografía son laboriosos y los resultados están limitados desde el punto de vista:

Dela taxonomía:a laespecieen concreto

- Del sistema productivo: cultivo, rotación, prácticas culturales, herbicidas, etc.

- De posibles interacciones: con otras especies y con el propio cultivo.

Planta Daninha, v. 12, n. 1, 1994. 
Además, en el medio de cultivo no existe una especie sola como población. En un estado más avanzado se realizará el estudio de varias poblaciones, compuestas por unas pocas especies que coexisten. Por lo tanto, no es posible dentro de 1a realidad cotidiana realizar un estudio detallado de las poblaciones de las malas hierbas más importantes, pero sí se pueden estudiar especies "tipo", o sistemas "tipo", desarrollar modelos y adaptarlos a otras especies de características parecidas, modificando los parámetros que intervienen en el modelo para cada una de esas especies.

\section{MODELOS POBLACIONALES Y RELACIONES CON LA PERDIDA DE RENDIMIENTO DEL CULTIVO}

Mortimer et al. (1989) han elaborado un modelo de dinámica de poblaciones de malas hierbas, partiendo de un modelo simple, y considerando en etapas sucesivas los efectos de la densidad de la población estudiada, la relación con el cultivo y los efectos del banco de semillas en suelo, la mezcla de especies, ]legando a enlazar el modelo de dinámica con los modelos de competición propuestos por Cousens (1985). Los pasos seguidos en esta propuesta se analizan a continuación:

\section{Modelo básico de dinámica de poblaciones}

El modelo más simple aplicable a una población de una especie única que ocupa un hábitat relaciona la población inicial $\mathrm{N}_{\mathrm{t}}$ en el momento t con la del ano siguiente $\mathrm{Nt}+\mathrm{i}$ mediante un coeficiente $\mathrm{k}$

$$
\mathrm{N}_{\mathrm{t}+1}=\mathrm{k} * \mathrm{~N}_{\mathrm{t}}
$$

Al cabo de $\mathrm{t}$ anos, si partimos de una población inicial No, la expresión sería:

$$
\mathrm{N}_{\mathrm{t}}=\mathbf{k}^{\mathrm{t} *} \mathrm{~N}_{\mathrm{o}}
$$

Cuando k = 1 la población está en equilibrio. Si k > 1 la población crece y si $\mathrm{k}<1$ la población desciende.

\section{Competencia intraespecífica}

Este modelo básico no considera muchos aspectos, como son la competencia intraespecífica entre los indivíduos de la especie, que hacen que el coeficiente $\mathrm{k}$ no sea una constante, sino que varíe en función de la densidad de plantas, de forma que cuando tas plantas están aisladas k es muy alto, y cuando la población es muy densa y ha alcanzado el equilibrio k toma el valor 1 .

En este caso la ecuación del modelo básico sería:

$$
\mathrm{N}_{\mathrm{t}+1}=\mathrm{F}\left(\mathrm{N}_{\mathrm{t}}\right) \mathrm{N}_{\mathrm{t}} \quad \mathrm{y} \quad \mathrm{k}=\mathrm{F}\left(\mathrm{N}_{\mathrm{t}}\right)
$$

Varios autores han obtendo estas relaciones. Para las malas hierbas Hassell (1975) propone:

$$
\mathrm{F}(\mathrm{N})=\mathrm{R}(1+\mathrm{a} N)^{-b}
$$

donde $\mathrm{R}$ es el incremento de la población cuando las plantas están poco agrupadas y no interfieren entre sí, y a y b son parámetros de la ecuación, característicos de la especie.
Para especies monocárpicas con generaciones discretas y semillas no persistentes en el suelo se puede expresar la dinámica de la población como:

$$
\mathrm{N}_{\mathrm{t}+1}=\mathrm{R} \mathrm{N}_{\mathrm{t}}\left(1+\mathrm{a} \mathrm{N}_{\mathrm{t}}\right)^{-\mathrm{b}}
$$

\section{Banco de semillas en el suelo}

Si consideramos además que una parte de las semillas del suelo no germinan y permanecen viables, Ia población de semillas en suelo $\mathrm{Nt}+\mathrm{i}$ se compondrá de dos fracciones: una correspondiente a la parte de la semilla del suelo que germina y otra que corresponde a la parte del banco de semilla que permanece en el suelo.

La ecuación se puede expresar:

$$
\mathrm{N}_{\mathrm{t}+1}=\mathrm{f} g \mathrm{~N}_{\mathrm{t}}\left(1+\mathrm{a} \mathrm{g} \mathrm{N}_{\mathrm{t}}\right)^{-\mathrm{b}}+\mathrm{s} \mathrm{N}_{\mathrm{t}}
$$

Siendo:

$\mathrm{f}=$ semilla producida por una planta que no está interaccionando con otras.

$\mathrm{s}=$ proporción de semillas del suelo que permanecen viables

y que se considera que es independiente de la densidad

$\mathrm{g}=$ proporción de semillas que germinan

$\mathrm{d}=$ proporción de semillas que mueren.

Tenemos: $\mathrm{s}+\mathrm{g}+\mathrm{d}=1$

La ecuación puede expresarse de forma simplificada:

$$
N_{t+1}=R^{\prime} N_{t}\left(1+a^{\prime} N_{t}\right)^{-b}+s N_{t}
$$

\section{Especies mezcladas}

En el caso de dos especies que interaccionan y pueden encontrarse en distintas densidades, el modelo indicado en el apartado 2 se transforma. Se parte de una nueva función en la que un individuo de un especie se expresa en términos equivalentes de la otra. En este caso:

$$
\mathrm{F}^{\prime}\left(\mathrm{N}_{1}\right)=\mathrm{F}\left(\mathrm{N}_{1}+\mathrm{h} \mathrm{N_{2 }}\right)
$$

Y las ecuaciones resultantes son:

$$
\begin{aligned}
& N_{1, t+1}=R_{1} N_{1, t}\left(1+a_{1}\left(N_{1, t}+h_{1} N_{2, t}\right)\right)^{-b_{1}} \\
& N_{2, t+1}=R_{2} N_{2, t}\left(1+a_{2}\left(N_{2, t}+h_{2} N_{1, t}\right)\right)^{-b_{2}}
\end{aligned}
$$

\section{Aplicaciones a la asociación cultivo y mala hierba.}

\section{- Caso de la interacción cultivo-mala hierba: Densidad de cultivo uniforme}

Si una de las especies que interrelacionan es el cultivo pueden aplicarse las ecuaciones anteriores. Pero si consideramos la densidad de cultivo constante las nuevas expresiones serían:

$$
\mathrm{N}_{\mathrm{t}+1}=\mathrm{R} \mathrm{N}_{\mathrm{t}}\left(1+\mathrm{a}\left(\mathrm{N}_{\mathrm{t}}+\mathrm{h}_{1} \mathrm{~N}_{\mathrm{c}}\right)\right)^{-\mathrm{b}}
$$

Pero en este caso N. es constante, y las ecuaciones pueden transformarse en:

$$
\mathrm{N}_{\mathrm{t}+1}=\mathrm{K}_{1} \mathrm{~N}_{\mathrm{t}}\left(1+\mathrm{K}_{2} \mathrm{~N}_{\mathrm{t}}\right)^{-\mathrm{b}}
$$

Esta expresión es idéntica a la correspondiente a especies solas y por tanto se puede usar esta aproximación y estudiar la dinámica de las malas hierbas sin incorporar 
explícitamente la densidad del cultivo, aunque $\mathrm{K}_{\mathrm{i}}$ y $\mathrm{K} 2$ no tienen un sentido biológico simple.

\section{- Pérdida de rendimiento debida a la presencia de malas hierbas}

Las ecuaciones anteriores para especies mezcladas pueden ser utilizadas en relación a la producción del cultivo. Así:

$$
\mathrm{N}_{\mathrm{c}, t+1}=\mathrm{R}_{\mathrm{c}} \mathrm{N}_{\mathrm{c}, \mathrm{t}}\left(1+\mathrm{a}_{\mathrm{c}}\left(\mathrm{N}_{\mathrm{c}, \mathrm{t}}+\mathrm{h}_{1} \mathrm{~N}_{\mathrm{t}}\right)\right)^{-\mathrm{b}_{\mathrm{c}}}
$$

Cuando $\mathrm{Nc}_{\mathrm{t}}$ es constante, Nsti4 resultaría ser el rendimiento del cultivo y la ecuación puede simplificarse:

$$
\mathrm{N}_{\mathrm{c}, \mathrm{t}+1}=\mathrm{C}_{1}\left(1+\mathrm{C}_{2} \mathrm{~N}_{\mathrm{t}}\right)^{-\mathrm{bc}}
$$

$\mathrm{C}_{1}$ es el rendimiento del cultivo libre de malas hierbas y sembrado a densidad Nc,t. La pérdida de rendimiento, Y, expresada en tanto por 1 , se define como:

$$
\begin{aligned}
& \mathrm{Y}=1-\left(1+\mathrm{C}_{2} \mathrm{~N}_{\mathrm{t}}\right)^{-\mathrm{b}_{\mathrm{c}}} \\
& \text { Cuando } \mathrm{b}_{\mathrm{c}}=1 \quad \mathrm{Y}=\mathrm{C}_{2} \mathrm{~N}_{\mathrm{t}}\left(1+\mathrm{C}_{2} \mathrm{~N}_{\mathrm{t}}\right)^{-1}
\end{aligned}
$$

que es una función hiperbólica de $\mathrm{N}_{\mathrm{t}}$ (Cousens, 1985)

\section{- Incorporación de prácticas de control}

Asumiendo que el control realizado es independiente de la densidad la expresión resultante sería:

$$
\mathrm{N}_{\mathrm{t}+1}=\mathrm{R} \mathrm{N} \mathrm{N}_{\mathrm{t}}\left(1+\mathrm{a} \mathrm{N}_{\mathrm{t}}\right)^{-\mathrm{b}}-\mathrm{T} \mathrm{N}_{\mathrm{t}}
$$

siendo $\mathrm{T}=\mathrm{d} * \mathrm{R}, \mathrm{y}$ los valores de $\mathrm{d}$, comprendidos entre $0 \mathrm{y}$ 1 , son una medida de la eficiencia del control.

\section{EL MODELO DEMOGRAFICO}

A partir del modelo básico de la dinámica de poblaciones:

$$
\mathrm{N}_{\mathrm{t}+1}=\mathbf{k} * \mathrm{~N}_{\mathrm{t}}
$$

se puede expresar el parámetro $\mathrm{k}$ en varias componentes que relacionan los estados funcionales. En el modelo demográfico resultante pueden ser considerados muy diversos aspectos según el ciclo de la especie que se esté estudiando. A continuación se detallarán algunos de ellos para los casos más frecuentes.

\section{Aspectos demográficos y semillas en el suelo}

El desarrollo del modelo básico se puede establecer haciendo consideraciones sucesivas. Considerando la tasa de crecimiento anual de la población independiente de la densidad y para el caso más sencillo de una especie monocárpica, a partir del contenido de semillas en el suelo, $\mathrm{k}$ se puede descomponer en dos sumandos:

$$
\mathrm{k}=\mathrm{A}+\mathrm{B}
$$

donde $\mathrm{A}=$. la fracción correspondiente a la semilla procedente de $\mathrm{N}_{\mathrm{t}}$ que germina y produce plantas y que puede expresarse como el producto de los parámetros demográficos genninación-emergencia (g), supervivencia (s) y fecundidad (f); y B es la proporción del banco de semillas la población $\mathrm{N}_{\mathrm{t}}$ que permanecen viables y en reposo en el suelo durante la estación t+1. Así el modelo resultaría:

$$
\mathrm{N}_{\mathrm{t}+1}=(\mathrm{g} * \mathrm{~s} * \mathrm{f}+\mathrm{B}) \mathrm{N}_{\mathrm{t}}
$$

Este modelo es útil para una primera aproximación, peno no considera algunos aspectos importantes que influyen considerablemente en la regulación de las poblaciones de malas hierbas. Entre estos aspectos está la emergencia de las plántulas en el tiempo, la cual afecta de forma considerable a la producción de semilla de ias distintas cohortes. En este caso para $\mathrm{z}$ cohortes la expresión matemática del modelo (Manlove et al., 1982) sería:

$$
\mathrm{N}_{\mathrm{t}+1}=\left(\sum_{\mathrm{i}=1}^{\mathrm{z}} \mathrm{g}_{\mathrm{i}} * \mathrm{~s}_{\mathrm{i}} * \mathrm{f}_{\mathrm{i}}+\mathrm{B}\right) \mathrm{N}_{\mathrm{t}}
$$

De la misma manera puede considerarse Ia influencia de la profundidad de enterramiento de la semilla, la cual puede influir sobre la producción final de semilla, bien sea porque la profundidad afecta a Ia periodicidad de emergencia, a la supervívencia de plántulas, o ai vigor y, en comecuencia, a Ia fecundidad.

Este tipo de modelos han sido utilizados por varios autores con distintos fines, tanto para analizar aspectos biológicos, tales como conocer los factores de mortalidad y reproducción o qué cohortes contribuyen más a mantener la población o aumentaria, como para determinar los momentos más adecuados para aplicar los herbicidas o el efecto de diferentes métodos de cultivo (Pollard, 1982; Wilson et al., 1984; Murdoch y Roberts, 1982; Manlove et al., 1982; Mortimer et al., 1980; Aarts, 1986; Fernandez-Quintanilla, 1988).

\section{Factores que afectan a los procesos demográficos}

Uno de los factores más importantes que influyen en la tasa final de crecimiento de una población es la densidad de plantas. Este aspecto puede ser considerado en cada uno de los procesos demográficos analizados, y más concretamente a la supervivencia de plántulas (s) y a la fecundidad (f).

En muchos casos, sobre todo cuando se trata de estudios básicos de dinámica o demografía, este aspecto no es considerado en toda su magnitud. Sin embargo, una población no crece indefinidamente, sino que en algún momento llega a un limite y se estabiliza. El modelo demográfico permite introducir este factor en aquellos procesos específicos que están influenciados por el tamano de la población.

Así, cada parámetro demográfico puede expresarse en función de la densidad y ser por tanto distinto para cada ano en lugar de ser una constante. Algunos autores han expresado la mortalidad según un modelo hiperbólico (Doyle et al., 1986; González-Andújar y Fernández-Quintanilla, 1991):

plantas adultas $(\mathrm{N})$

$$
\mathrm{N}=\mathrm{P}(1+\mathrm{uP})-{ }^{1} \text { para el paso de plántulas }(\mathrm{P}) \text { a }
$$

y la producción de semillas, fecundidad (f), en función de la fecundidad de una planta aislada $(\mathrm{F})$ como:

$$
\mathrm{f}=\mathrm{F}(1+\mathrm{vN})^{-1}
$$

u y v son parámetros de la ecuación, característicos de cada especie. 
Otro factor importante que puede considerarse en el modelo demográfico es la densidad del cultivo. Este factor suele considerarse en términos de equivalencia entre plantas de cultivo y plantas de la especie considerada. Así Firbank y Watkinson (1985) modifican y expresan las ecuaciones anteriores como:

$$
\mathrm{N}=\mathrm{P}(1+\mathrm{u}(\mathrm{P}+\mathrm{rC}))^{-1} \quad \mathrm{f}=\mathrm{F}(1+\mathrm{v}(\mathrm{N}+\mathrm{sC}))^{-k}
$$

siendo: u, r, v, s y k parámetros de la ecuación y C la densidad de cultivo.

Otros autores utilizan otras funciones diferentes: logarítmicas, exponenciales, etc. Los casos descritos son frecuentes en la literatura, peru también cualquier otro factor que influya en un determinado proceso puede ser considerado dentro del modelo demográfico, haciendo el parámetro demográfico función del factor considerado por medio de una función matemática, o de una tasa constante, como por ejemplo la tasa de mortalidad debida a la aplicación de un herbicida o el efecto de una escarda manual.

3. Modelos demográficos y pérdidas de cosecha

De la misma manera que para el modelo poblacional, es posible establecer relaciones entre el modelo demográfico y la pérdida de cosecha producida por una determinada mala hierba.

Firbank y Watkinson (1986) analizan, utilizando el modelo demográfico, la dinámica de Agrostemma githago a lo largo de los anos, para distintas eficacias de control, al tiempo que determinan la producción de semillas de trigo para las distintas densidades de infestación. En este modelo se utiliza la biomasa como parámetro de enlace entre la producción de semillas y el número de plantas, tanto para la mala hierba, como para el cultivo.

\section{MODELOS MATRICIALES}

En los casos en que el factor tiempo es influyente dentro de un ciclo anual de la mala hierba, la expresión matricial de los procesos facilita los cálculos. Este es el caso por ejemplo de los estudios de las diferentes cohortes de la mala hierba en los estudios de dinámica, los estudios de supervivencia y los de emergencia de semillas en el suelo. De la misma manera se requiere un modelo matricial para

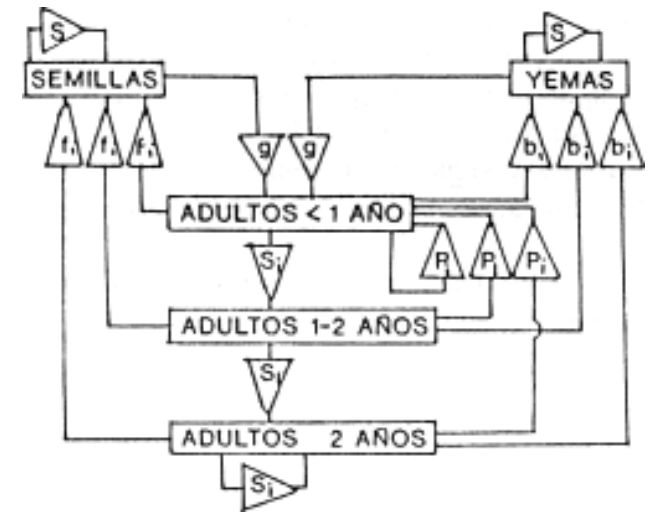

\begin{tabular}{|c|c|c|c|c|c|}
\hline & $\mathrm{AC}$ & $\begin{array}{l}\text { dulto } \\
<1\end{array}$ & $\begin{array}{l}\text { Adulto } \\
1-2\end{array}$ & $\begin{array}{l}\text { Adulto } \\
>2\end{array}$ & Yema \\
\hline Semilla & 0.07 & 9.52 & 13.9 & 15.9 & 0 \\
\hline Adulto $<1$ & 0.0001 & 1.96 & 1.96 & 1.96 & 0.01 \\
\hline Adulto $1-2$ & 0 & 0.86 & 0 & 0 & 0 \\
\hline Adulto $>2$ & 0 & 0 & 0.47 & 0.3 & 0 \\
\hline Yema & 0 & 50 & 50 & 50 & 0.94 \\
\hline
\end{tabular}

estudiar la dinámica de aquellas especies cuya población no puede cuantificarse a partir de un estado funcional sólamente, como es el caso de especies perennes que podemos tener simultáneamente en estado de semilla, propágulo vegetativo, planta inmadura, planta fértil, etc.

El modelo matricial es fácil de usar con ayuda de ordenadores y permite también considerar los efectos de la densídad de cultivo, densidad de la mala hierba, etc.

Ejemplos de uso del modelo matricial los encontramos en estudios de dinámica de Avena sterilis (González-Andújar et al., 1986), en los estudios de distribución de semillas en suelo (Cousens y Moss, 1990), o para predecir las infestaciones de Agropyron repens (MacMahon y Mortimer, 1980; Mortimer, 1983). En la figura 8 se exponen parte de los resultados de este último trabajo: el ciclo de Agropyron repens y la matriz de transición para dos anos consecutivos.

\section{ANALISIS DE SENSIBILIDAD Y VALIDACION DEL MODELO}

Una de las preguntas que nos hacemos acerca del modelo demográfico es qué componentes de la población contribuyen más al incremento de la misma. La sensibilidad de la tasa de crecimiento de la población a los cambios producidos en uno de sus componentes es:

\section{$\delta \mathrm{k} / \delta \mathrm{a}$}

siendo $\mathrm{k}$ la tasa de crecimiento de la población y a un elemento de transición entre estados, o un elemento de la matriz de transición.

De forma simplificada puede expresarse en términos de incrementos:

$$
\frac{\Delta \text { output/output }}{\Delta \text { input/input }}
$$

Este coeficiente de sensibilidad nos indica, por un lado, cual es el proceso en el que debemos actuar para regular la población y, también, cual es el dato que es necesario determinar con más precisión para que los resultados sean acordes con la realidad.

La validación del modelo en condiciones de campo es imprescindible para poder utilizarlo en situaciones diferentes como herramienta predictiva y, por supuesto, para justificar

FIG. 8 - Demografia de Agropyron repens y matriz de transición. (Mortimer, 1983). 
la utilidad del trabajo realizado. La validación puede hacerse con pmcedimientos matemáticos simples como es el cálculo $\mathrm{x}^{2}$ entre valores calculados con el modelo y valores observados. Actualmente hay muy pocos modelos validados (González-Andújar, 1991).

\section{COMENTARIOS}

Los modelos de dinâmica y demografía de malas hierbas realizados hasta el momento tienen un valor fundamentalmente cualitativo. Además, los trabajos en malherbología se realizan muchas veces en condiciones de campo bastante restringidas, por lo que se hace más necesario aún validar muchos de los modelos establecidos. No obstante, en otras disciplinas los modelos están siendo aplicados con gran eficacia y en el caso de la malherbologla hay aún mucho trabajo por hacer.

La integración de modelos demográficos, modelos de competencia, métodos de control y toma de decisiones permitirán en un futuro, esperemos próximo, establecer sistemas más racionales de control de malas hierbas y disminuir los costes de cultivo y los riesgos de contaminación ambiental.

\section{LITERATURA CITADA}

AARTS, H.F.M. A computerized model for predicting changes in a population of Galium aparine. In: SYMPOSIUM ON ECONOMICS WEED CONTROL, 1986. Proceedings. EWRS, 1986. p.277-284.

COUSENS, R.D. A simple model relating yield loss to weed density. Annals of Applied Biology, v.107, p.239252,1985 .

COUSENS, R.D. The use of population models in the study of the economics of weed control. In: SYMPOSIUM ON ECONOMICS WEED CONTROL, 1986. Proceedings. EWRS, 1986. p.269-276.

COUSENS, R.; MOSS, S.R. A model of the effects of cultivation on the vertical distribution of weed seeds within the soil. Weed Research, v.30, p.61-70, 1990.

DOYLE, C.J., COUSENS, R.; MOSS, S.R. A model of the economics of controlling Alopecurus myosuroides Huds. in winter wheat. Crop Protection, v.5, p.143150, 1986.

FERNANDEZ-QUINTANILLA, C. Studying the population dynamics of weeds. Weed Research, v.28, p.443447, 1988.

FERNANDEZ-QUINTANILLA， C., NAVARRETE， L.; TORNER, C. The influence of crop rotation on the population dynamics of Avena sterilis subsp. ludoviciana in Central Spain. In: SYMPOSIUM ON WEED PROBLEMS IN THE MEDITERRANEAN AREA, 1, 1984. Proceedings. EWRS, 1986. p.9-16.
FERNANDEZ-QUINTANILLA， C. Dinâmica de las poblaciones de malas hierbas. Internacional Course on Weed Biology and Control for Mediterranean Conditions. Instituto Agronómico Mediterrâneo de Zaragoza (I.A.M.Z.). International Center for Ad vanced Mediterranean Agronomic Studies (C.I.H.E. A.M.), 1990.

FIRBANK, L.G.; WATKINSON A.R. On the analysis of competition within two-species mixtures of plants. Journal of Applied Ecology, v.22, p.503-517, 1985.

FIRBANK, L.G. y WATKINSON A.R. Modelling the population dynamics of an arable weed and its effects upon crop yield. Journal of applied Ecology, v.23, p.147-159, 1986.

FR ANCE, J. Mathematical modelling in agricultural science. Weed Research, v.28, p.419-423, 1988.

GONZALEZ-ANDUJAR, J.L. Modelización de la ecología y el control de las malas hierbas. (En preparación). 1991.

GONZALEZ-ANDUJAR, J.L., FERNANDEZ, A.; FERNANDEZ-QUINTANILLA, C. Modelización y simulación de la dinámica de poblaciones de plantas mediante el modelo matricial de Leslie. Investigación Agraria, Serie. Producción y Protección Vegetales, v.1, n.2, p.209-218, 1986.

GONZALEZ -ANDUJAR, J.L.; FERNANDEZ QUINTANILLA, C. Modelling the population dynamics of Avena sterilis under dry-land cereal cropping system. Journal of Applied Ecology, v.28, p.1627, 1991.

JIMENEZ-HIDALGO, M., SAAVEDRA, M.; GARCIATORRES, L. Notas sobre dinâmica de poblaciones de Phalaris brachystachys y P. paradoxa en una rotación trigo-girasol. In: Reunión de la SOCIEDAD ESPANOLA DE MALHERBOLOGÍA, 1990. Actas. p.299306.

LOPEZ-GRANADOS, F.; GARCIA -TORRES, L. Evolución de las poblaciones de Orobanche crenata Forsk en habas Vicia faba L. In: Reunión de la SOCIEDAD ESPANOLA DE MALHERBOLOGÍA, 1990. Actas. p.285-289.

MANLOVE, R.J., MORTIMER, A.M.; PUTWAIN, P.D. Modelling wild oat populations and their control. In: BRITISH CROP PROTECTION CONFERENCEWEEDS, 1982. Proceedings. p. 749-756.

MCMAHON, D.J.; MORTIMER, A.M. The prediction of couch infestations - A modelling approach. In: BRITISH CROP PROTECTION CONFERENCE-WEEDS, 1980. Proceedings. p.601-607.

MORTIMER, A.M. On weed demography. In: Fletcher, W.W. Recent Advances in Weed Research. Commonwealth Agricultural Bureaux, 1983. p. 3-40.

MORTIMER, A.M. MACMAHON, DJ., MANLOVE, R.J.; PUTWAIN, P.D. The prediction of weed infestations and cost of difering control strategies. In: BRITISH CROP PROTECTION CONFERENCE-WEEDS, 1980. Proceedings. p.415-422.

MORTIMER, A.M., SUTTON, JJ.; GOULD, P. On robust weed population models. Weed Research, v.29, p.229-238, 1989. 
MURDOCH, A.J.; ROBERTS, E.H. Biological and financial criteria of long-term control strategies for annual weeds. In: BRITISH CROP PROTECTION CONFERENCE-WEEDS, 1982. Proceedings. p.741-748.

PALMA, V., SAAVEDRA, M.; GARCIA-TORRES, L. Dinámica de poblaciones de Avena sterilis subsp. sterilis L. en trigo (Triticum aestivum L.). In: Reunión de la SOCIEDAD ESPANOLA DE MALHERBOLOGÍA, 1990. Actas. p.285-289.

POLLARD, F. A computer model for predicting changes in a population of Bromus sterilis in continuos winter cereals. In: BRITISH CROP PROTECTION CONFERENCE-WEEDS, 1982. Proceedings. p.973-982.
SAGAR, G.R.; MORTIMER, A.M.. An approach to the study of the population dynamics of plants with special reference to weeds. Applied Biology, p. 1-47, 1976.

WILSON, B.J. COUSENS, R.; CUSSANS, G.W. Exercises in modelling population of A vena fatua L. to aid strategic planinning for the long term control of this weed in cereals. In: INTERNATIONAL SYMPOSIUM ON WEED BIOLOGY, ECOLOGY AND SYSTEM ATIC, 7., 1984. p.287-294. 